\title{
COMPORTAMENTO OPERACIONAL DE ASSOCIAÇÕES ENTRE SULCADORES E DISCOS DE CORTE PARA SISTEMA DE SEMEADURA DIRETA
}

Doi:http://dx.doi.org/10.1590/1809-4430-Eng.Agric.v35n3p 542-554/2015

\section{TIAGO R. FRANCETTO ${ }^{1}$, AIRTON DOS S. ALONÇO ${ }^{2}$, MATEUS P. BELLÉ ${ }^{3}$, CRISTIAN J. FRANCK ${ }^{4}$, DAUTO P. CARPES ${ }^{5}$}

\begin{abstract}
RESUMO: O objetivo foi avaliar o desempenho operacional quanto ao requerimento de tração, consumo energético e eficiência de trabalho de associações entre elementos de corte dos resíduos culturais e de rompedores do solo, em função da velocidade de deslocamento. Este trabalho foi realizado em propriedade agrícola situada no município de Santa Maria (RS), em solo classificado como Argissolo Vermelho e textura francoarenosa. O experimento foi composto por 24 combinações de tratamentos, em um esquema fatorial de $2 \times 3 \times 4$. Estes foram obtidos pela interação dos fatores sulcadores (haste e disco), discos de corte (sem disco, liso e ondulado) e velocidades $\left(1,11 ; 1,67 ; 2,22\right.$ e $\left.2,78 \mathrm{~m} \mathrm{~s}^{-1}\right)$. Os dados de desempenho do trator foram adquiridos com a utilização de instrumentação eletrônica. As associações entre sulcador e disco apresentaram melhor desempenho nas velocidades de 1,11 e $1,67 \mathrm{~m} \mathrm{~s}^{-1}$ por exigirem menor força de tração e consumo de combustível. Além disso, o uso de discos permitiu uma redução da ampliação da força de tração quando a velocidade passou de 1,11 para $2,78 \mathrm{~m} \mathrm{~s}^{-1}$. A haste exigiu maior demanda de tração do que os discos desencontrados, e ambos, ao serem combinados com os discos de corte, tiveram suas demandas aumentadas. Outrossim, a mobilização do solo não foi influenciada pela velocidade.
\end{abstract}

PALAVRAS-CHAVE: Semeadora-adubadora, Relação máquina-solo, Ensaio de máquinas agrícolas, Haste sulcadora, Discos duplos desencontrados, Mecanismo de corte.

\section{OPERATING PERFORMANCE OF FURROWERS AND CUTTING DISKS ASSOCIATION FOR NO-TILLAGE SYSTEM}

\begin{abstract}
The objective was to evaluate tractor operating performance regarding traction application, energy consumption and working efficiency in association of crop residue cutting implements and soil breakers as the travelling speed. Experiment was performed at a farm in Santa Maria (RS), Brazil. Local soil was classified as Red Ultisol with sandy loam texture. The experiment consisted of 24 treatment combinations in a $2 \times 3 \times 4$ factorial scheme. The combinations were composed by interactions furrowers (furrow opener fixed and rotary), cutting disks (without disk, plain disk, and undulating disk) and travelling speeds $\left(1.11,1.67,2.22\right.$ and $\left.2.78 \mathrm{~m} \mathrm{~s}^{-1}\right)$. Tractor performance data were acquired through electronic instrumentation. Furrower and cutting disk association had improved performance at speeds of 1.11 and $1.67 \mathrm{~m} \mathrm{~s}^{-1}$ for requiring less traction and fuel consumption. Furthermore, disks reduced required traction at speed range of 1.11 to $2.78 \mathrm{~m}$ $\mathrm{s}^{-1}$. On the other hand, furrow opener device demanded higher tractions than mismatched disks; and both of them combined with cutting disks had increased demand too. Likewise, soil mobilization soil was not influenced by speed.
\end{abstract}

KEYWORDS: Row crop planter, Machine-soil relationship, Agricultural machinery testing, Furrow opener drills, Mismatched double disks, Cutting mechanism.

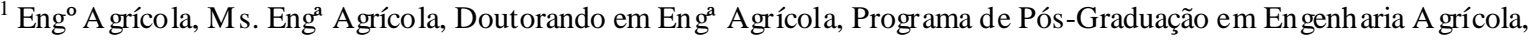
Universidade Federal de Santa Maria/Santa Maria - RS, tiagofrancetto@gmail.com

${ }^{2}$ Eng $^{\mathbf{o}}$ A grícola, Prof. Doutor, Departamento de Engenharia Rural, Universidade Federal de Santa Maria/Santa Maria - RS, alonco@ccr.ufsm.br

${ }^{3}$ Eng $^{\circ}$ A grônomo, Ms. Eng ${ }^{\mathbf{a}}$ Agrícola, Doutorando em Eng $g^{\mathbf{a}}$ Agrícola, Programa de Pós-Graduação em En genh aria Agrícola, Universidade Federal de Santa Maria/Santa Maria - RS, mateuspotrichbelle@y ahoo.com.br

${ }^{4}$ Eng $^{\circ}$ A grícola, Ms. Eng ${ }^{a}$ Agrícola, Programa de Pós-Graduação em Engenharia Agrícola, Universidade Federal de Santa Maria/Santa Maria - RS, cristianjfranck@gmail.com

${ }^{5}$ Eng $^{\circ}$ A grônomo, Ms. Eng ${ }^{\mathbf{a}}$ Agrícola, Doutorando em Eng ${ }^{\mathbf{a}}$ Agrícola, Programa de Pós-Graduação em En genh aria A grícola, Universidade Federal de Santa Maria/Santa Maria-RS, dautocarpes@gmail.com 


\section{INTRODUÇÃO}

A expansão da agricultura brasileira deve-se, em grande parte, ao aumento da área cultivada no decorrer dos últimos 40 anos. O País passou de aproximadamente 40 milhões de hectares em 1980, para 50 milhões de hectares em 2013, segundo o Instituto Brasileiro de Geografia e Estatística (IBGE, 2013), permitindo um aumento substancial da produção agrícola. Contudo, apenas o incremento da área não é suficiente para que se busque a ampliação da produção.

A produção nacional de grãos passou de valores inferiores a 60 milhões de toneladas em 1980, para índices superiores a 180 milhões de toneladas em 2012 (IBGE, 2013). Entretanto, a área total de produção dessas culturas manteve-se em aproximadamente 40 milhões de hectares. Dessa forma, o emprego de novas tecnologias e técnicas tem demonstrado cada vez mais sua importância no aumento da produção agrícola brasileira.

Nesse contexto, recebe destaque a mecanização agrícola que, através do desenvolvimento de equipamentos mais modernos, tem permitido, além do aumento da produção, o acréscimo da capacidade operacional nas mais distintas atividades. Contudo, tendo em vista que o uso de máquinas implica grande parte dos custos totais de produção, devem-se procurar alternativas que proporcionem a otimização do uso ligado à ampliação da vida útil, na busca pela melhoria do aproveitamento dos recursos produtivos e, consequentemente, na redução dos custos da atividade.

Todavia, como a agricultura se apresenta ligada a diversos fatores externos, como o clima, muitas vezes o intervalo disponível para a realização de uma determinada tarefa é reduzido. Dentre as mais distintas atividades na agricultura, que empregam o uso de máquinas e dependem desse tempo, tem-se a semeadura, que representa uma das mais importantes etapas da produção agrícola pelo fato de propiciar o estabelecimento da cultura.

Semear é uma técnica milenar e, de sua qualidade, dependem o sucesso e a produtividade de uma cultura agrícola. Os aspectos mais relevantes para isso estão relacionados com o desempenho da semeadora-adubadora no que se refere ao corte eficiente dos resíduos culturais, à abertura do sulco e à colocação da semente e do fertilizante em profundidades corretas e em contato com o solo.

O mecanismo de corte de palha é encontrado em diferentes diâmetros e formatos, de forma que tais variações podem promover o cisalhamento dos resíduos vegetais e a abertura de uma pequena fissura no solo com distinta eficiência e variações significativas na qualidade da operação.

A abertura do sulco, processo subsequente ao corte da palha, pode ser promovida pela utilização de discos duplos ou de hastes sulcadoras. Contudo, MIALHE (2012) destaca o fato de que, em semeadoras de semeadura direta, as características relativas ao comportamento dos abridores de sulco, de forma isolada dos demais componentes, são relativamente pouco estudadas.

Desse modo, o objetivo geral deste trabalho foi avaliar o desempenho operacional do conjunto trator/porta-ferramentas equipado com diferentes configurações dos elementos de corte dos resíduos culturais e rompimento do solo, em função da velocidade de deslocamento.

\section{MATERIAL E MÉTODOS}

O experimento foi realizado em propriedade agrícola situada no município de Santa Maria (RS). As coordenadas geográficas do local são 2954'08" latitude sul e 5349'39" longitude oeste, com altitude média $97 \mathrm{~m}$ acima do nível do mar. Foi observada a existência de palha de soja (Glycine max) e de azevém (Lolium multiflorum), além de algumas plantas daninhas. Foi verificada a presença de $287,20 \mathrm{~g} \mathrm{~m}^{-2}$ de matéria seca sobre a superfície do solo por meio do emprego do método da estufa. A área é utilizada sucessivamente para a produção da cultura da soja, como cultura de verão, sendo usada no inverno como local de criação de gado para pecuária de corte.

A caracterização física do solo foi realizada por meio da coleta de amostras a campo, em profundidade de 0 a $0,20 \mathrm{~m}$, seguindo metodologia proposta pela EMBRAPA (1997), para determinações da densidade do solo e do teor de água. Para a primeira, foi verificado o valor médio de $1,64 \mathrm{~g} \mathrm{~cm}^{-3}$, enquanto a segunda permaneceu em 13,15\%. A caracterização da textura do solo 
consistiu na adoção do método de vettori. Esta é composta por $17,59 \%$ de argila, $28,44 \%$ de silte e $53,97 \%$ de areia, sendo o solo classificado texturalmente como francoarenoso. Este foi classificado como Argissolo Vermelho. A resistência à penetração do solo foi determinada com a utilização de um penetrômetro eletrônico, marca Falker, modelo PLG 1020, obtendo o valor médio de $1.220 \mathrm{kPa}$. A coleta de dados foi realizada na profundidade de 0 a $0,40 \mathrm{~m}$, com aquisição de um dado a cada $0,010 \mathrm{~m}$ de profundidade.

O trator utilizado para tracionar o porta-ferramentas foi um New Holland TL75E Exitus 4x2, com massa de embarque de $3.390 \mathrm{~kg}$. Durante o experimento, a TDA esteve desligada com o trator operando em cond ições normais, sem o bloqueio do diferencial acionado. A pressão interna do pneu dianteiro (12.4-24) foi de 190,0 $\mathrm{kPa}$, e do traseiro (18.4-30), de $180,0 \mathrm{kPa}$.

Os elementos de abertura de sulco utilizados foram uma haste sulcadora (SF) e discos duplos desencontrados (SR). O primeiro apresenta um ângulo de ataque de $55^{\circ}$, espessura da ponteira de 20 $\mathrm{mm}$ e da haste de $10 \mathrm{~mm}$. Já o segundo exibe um diâmetro de $390 \mathrm{~mm}$, ângulo entre os planos de rotação dos discos de $12^{\circ}$ e altura do ponto de contato de $70 \mathrm{~mm}$. A profundidade de trabalho foi de $0,12 \mathrm{~m}$ para o primeiro e de 0,06 para o segundo.

Os discos de corte empregados foram um liso (DL) e um ondulado (DO) com 20 ondas, além da cond ição sem disco (SD). A mbos possuem diâmetro de $460 \mathrm{~mm}$ e trabalharam à profund id ade de corte de $50 \mathrm{~mm}$.

Os tratamentos foram compostos pela interação dos fatores mecanismos de abertura de sulco (SF e SR), corte de resíduos culturais (SD, DL e DO) e velocidades de deslocamento $(1,11 ; 1,67$; 2,22 e $2,78 \mathrm{~m} \mathrm{~s}^{-1}$ ). O delineamento experimental utilizado foi o de blocos ao acaso, constituído por 24 tratamentos, com três repetições em cada, em esquema fatorial de $2 \times 3 \times 4$, em área total de $4.320,00 \mathrm{~m}^{2}$. As parcelas, distribuídas nesta última, tiveram $180,00 \mathrm{~m}^{2}$, com 3,0 m de largura por 60,0 m de comprimento.

Utilizou-se de um datalogger marca Campbell Scientific, modelo CR 1000 para armazenamento dos dados gerados pelos sensores de rotação (determinação indireta do patinamento), célula de carga (caracterização da demanda de tração) e fluxômetro (aferição do consumo horário de combustível).

O patinamento dos rodados motrizes do trator foi determinado com o auxílio de sensores indutivos de medição de rotação. Estes permaneceram fixos em um suporte ligado ao para-lama do trator, enquanto uma engrenagem, com 40 dentes, presa em um suporte acoplado ao aro dos pneus traseiros, girava juntamente com o rodado. Cada engrenagem possui um sensor de proximidade que emite pulsos elétricos de acordo com a passagem dos dentes, indicando a frequência da rotação. Dessa maneira, conforme a [eq. (1)] é possível calcular a velocidade da roda que desenvolve esforço tratório.

$$
\text { Vroda }=\frac{(\mathrm{F} \times \mathrm{P} \times 3,6)}{\mathrm{nd}}
$$

em que,

Vroda - velocidade da roda $\left(\mathrm{km} \mathrm{h}^{-1}\right)$;

F - frequência captada pelo sensor $(\mathrm{Hz})$;

$\mathrm{P}$ - perímetro da roda $(\mathrm{m})$,

nd - número de dentes da engrenagem.

De posse da informação da velocidade média de deslocamento do conjunto, obtida por uma roda odométrica que não desenvolve esforço tratório, e da velocidade da roda, obtém-se o valor de patinamento, utilizando a [eq. (2)].

$$
\mathrm{Pa}=\frac{(\text { Vroda }- \text { VSG })}{\text { Vroda }} \times 100
$$


em que,

$\mathrm{Pa}$ - patinamento $(\%)$;

Vroda - velocidade da roda que desenvolve esforço $\left(\mathrm{km} \mathrm{h}^{-1}\right)$,

VSG - velocidade da roda que não desenvolve esforço $\left(\mathrm{km} \mathrm{h}^{-1}\right)$.

Uma célula de carga tipo $\mathrm{S}$, de cinco toneladas (t), marca Berman, foi empregada para a determinação da força de tração requerida. Esta esteve acoplada em um suporte retrátil fixo à barra de tração e ao cabeçalho do porta-ferramentas. O implemento demandou uma força para seu funcionamento, denominada resistência ao rolamento, e esta foi medida em igual amplitude, através de sinais elétricos emitidos de acordo com a intensidade e descontados do valor final. Estes sinais, gerados em milivolts $(\mathrm{mV})$, foram convertidos e armazenados no datalogger.

A força de tração média foi determinada com base na [eq. (3)], utilizando os dados da força de tração instantânea e da resistência ao rolamento.

$$
\mathrm{Ft}=\mathrm{Fi}-\mathrm{Rr}
$$

em que,

Ft - força de tração média $(\mathrm{kN})$;

Fi - força de tração instantânea $(\mathrm{kN})$,

$\mathrm{Rr}$ - resistência ao rolamento $(\mathrm{kN})$.

A partir do valor da força média de tração, foi possível calcular a potência disponível na barra de tração, conforme a [eq. (4)], possibilitando o cálculo do consumo específico de combustível.

$$
\mathrm{Pb}=\mathrm{Ft} \times \mathrm{V}
$$

em que,

$\mathrm{Pb}$ - potência na barra de tração $(\mathrm{kW})$;

Ft - força de tração média $(\mathrm{kN})$,

$\mathrm{V}$ - velocidade média de deslocamento do conjunto $\left(\mathrm{m} \mathrm{s}^{-1}\right)$.

A resistência específica operacional foi obtida pela razão entre a força de tração média e a área mobilizada, segundo a [eq. (5)].

$$
\operatorname{Reo}=\frac{\mathrm{Ft}}{\mathrm{Am}}
$$

em que,

Reo - resistência específica operacional $\left(\mathrm{kN} \mathrm{m}^{-2}\right)$;

$\mathrm{Ft}$ - força de tração média $(\mathrm{kN})$,

Am - área de solo mobilizada $\left(\mathrm{m}^{2}\right)$.

Para a aferição do consumo horário de combustível, fez-se uso de um fluxômetro da marca Oval M-III, modelo LSF41, composto por duas engrenagens. Com base na rotação destas, o equipamento emite pulsos elétricos que são armazenados pelo datalogger, de forma que, com a utilização da [eq. (6)], calcula-se o consumo em litros por hora.

$$
\mathrm{Ch}=\mathrm{Pu} \times 1,8
$$


em que,

Ch - consumo horário de combustível $\left(\mathrm{L} \mathrm{h}^{-1}\right)$;

$\mathrm{Pu}$ - pulsos elétricos gerados pelo fluxômetro.

Por sua vez, o consumo específico de combustível foi obtido empregando-se a [eq. (7)]. Este é alcançado pela relação entre o produto da densidade do combustível e o do consumo horário pela potência na barra de tração.

$$
\mathrm{Ce}=\frac{(\mathrm{D} \times \mathrm{Ch})}{\mathrm{Pb}}
$$

em que,

Ce - consumo específico de combustível $\left(\mathrm{g} \mathrm{kWh}^{-1}\right)$;

D - densidade do combustível $\left(\mathrm{g} \mathrm{L}^{-1}\right)$;

Ch - consumo horário de combustível $\left(\mathrm{L} \mathrm{h}^{-1}\right)$,

$\mathrm{Pb}$ - potência na barra de tração $(\mathrm{kW})$.

O consumo específico operacional é adquirido pela razão entre o consumo específico e a área mobilizada, segundo a [eq. (8)].

$$
\mathrm{Ceo}=\frac{\mathrm{Ce}}{\mathrm{Am}}
$$

em que,

Ceo - consumo de combustível específico operacional $\left(\mathrm{g} \mathrm{kWh}^{-1} \mathrm{~m}^{-2}\right)$;

Ce - consumo específico $\left(\mathrm{g} \mathrm{kWh}^{-1}\right)$,

Am - área mobilizada de solo $\left(\mathrm{m}^{2}\right)$.

$\mathrm{Na}$ área experimental, foram realizadas três leituras para a avaliação da área elevada e mobilizada de solo. A primeira foi efetuada antes da passagem do porta-ferramentas, a segunda e a terceira após a passagem deste no mesmo local, obtendo a forma geométrica do sulco. Para garantir que as amostragens fossem realizadas no mesmo local, utilizou-se de duas estacas de madeira, uma de cada lado do local previs to para a abertura do sulco. Os perfis do solo foram marcados em folhas A2 de papel milimetrado, com o uso de canetas, traçando uma linha entre as extremidades dos pontos coletados em cada folha, os quais forneceram o perfil natural do solo, área elevada e mobilizada.

Primeiramente o perfilômetro foi posicionado transversalmente ao futuro sulco para obter-se o perfil natural do solo, anotando-se os dados. Realizou-se então a passagem do porta-ferramentas e posicionou-se novamente o aparelho no local anteriormente utilizado, com a finalidade de se fazer o registro do perfil após a passagem do sulcador. Por fim, removeu-se manualmente todo o solo mobilizado do sulco até a profundidade onde o mesmo não foi movimentado, tomando-se o cuidado de não alterar o perfil para a obtenção do perfil de solo mobilizado subsuperficialmente.

Posteriormente, esses gráficos foram fotografados, ficando a máquina fixa (posição x, y e z). Em seguida, estas foram inseridas no programa computacional Auto Cad para traçar as linhas de contorno dos perfis e, através da utilização de ferramentas para leitura de área, determinou-se a área em metros quadrados. A diferença entre a primeira e a terceira leitura forneceu a área mobilizada de solo (Am), enquanto a diminuição desse valor na área da segunda leitura indicou área elevada (Ae).

Após a aquisição dos dados de desempenho, eles foram submetidos a análises de variância, utilizando o teste de análise de Tukey, ao nível de $5 \%$ de probabilidade de erro. Foi testada a normalidade dos dados, utilizando o teste de Kolmogorov-Smirnov, e a homogeneidade de variâncias pelo teste de Cochran. Para estas, utilizou-se do software Assistat 7.6 beta 2012. 


\section{RESULTADOS E DISCUSSÃO}

A análise de variância (ANOVA) das variáveis, com suas respectivas médias, níveis e os resultados dos testes $\mathrm{F}$ são apresentados na Tabela 1. Foram observadas a normalidade dos dados e a homo geneidade de variâncias.

TABELA 1. Síntese da análise estatística de variância com as médias dos fatores, seus níveis e os resultados do teste $\mathrm{F}$. Variance analysis synthesis with factor averages, levels and F test results.

\begin{tabular}{|c|c|c|c|c|c|c|c|c|}
\hline \multirow[b]{2}{*}{ Fatores } & \multicolumn{8}{|c|}{ Variáveis } \\
\hline & $\begin{array}{l}\mathrm{Pa} \\
(\%)\end{array}$ & $\begin{array}{l}\mathrm{Am} \\
\left(\mathrm{m}^{2}\right)\end{array}$ & $\begin{array}{c}\text { Ceo } \\
\left(\mathrm{g} \mathrm{kWh}^{-1} \mathrm{~m}^{-2}\right)\end{array}$ & $\begin{array}{c}\mathrm{Ft} \\
(\mathrm{kN})\end{array}$ & $\begin{array}{c}\mathrm{Ch} \\
\left(\mathrm{Lh}^{-1}\right)\end{array}$ & $\begin{array}{c}\mathrm{Ce} \\
\left(\mathrm{g} \mathrm{kWh}^{-1}\right)\end{array}$ & $\begin{array}{l}\mathrm{Ae} \\
\left(\mathrm{m}^{2}\right)\end{array}$ & $\begin{array}{c}\text { Reo } \\
\left(\mathrm{kN} \mathrm{m}^{-2}\right)\end{array}$ \\
\hline \multicolumn{9}{|l|}{$\overline{\text { SULCADORES }}$} \\
\hline Fixo (SF) & $3,29 \mathrm{a}$ & $0,0126 \mathrm{a}$ & $75,03 \mathrm{~b}$ & $2,14 \mathrm{a}$ & $3,75 \mathrm{a}$ & $0,92 \mathrm{~b}$ & $0,0052 \mathrm{a}$ & $170,51 \mathrm{~b}$ \\
\hline Rotativo (SR) & $3,15 \mathrm{~b}$ & $0,0096 \mathrm{~b}$ & $132,60 \mathrm{a}$ & $1,75 \mathrm{~b}$ & $3,61 \mathrm{~b}$ & $1,26 \mathrm{a}$ & $0,0028 \mathrm{~b}$ & $187,46 \mathrm{a}$ \\
\hline \multicolumn{9}{|l|}{$\begin{array}{l}\text { DISCOS DE } \\
\text { CORTE }\end{array}$} \\
\hline$\overline{\text { Sem disco (SD) }}$ & $2,89 \mathrm{~b}$ & $0,0108 \mathrm{~b}$ & $126,14 \mathrm{a}$ & $1,66 \mathrm{c}$ & $3,73 \mathrm{a}$ & $1,31 \mathrm{a}$ & $0,0049 \mathrm{a}$ & $156,19 b$ \\
\hline Disco liso (DL) & $3,34 \mathrm{a}$ & $0,0106 \mathrm{~b}$ & $84,90 \mathrm{~b}$ & $2,04 \mathrm{~b}$ & $3,66 \mathrm{a}$ & $0,85 \mathrm{c}$ & $0,0040 \mathrm{ab}$ & $198,15 \mathrm{a}$ \\
\hline $\begin{array}{l}\text { Disco ondulado } \\
\text { (DO) }\end{array}$ & $3,42 \mathrm{a}$ & $0,0119 \mathrm{a}$ & $100,39 b$ & $2,13 \mathrm{a}$ & $3,66 \mathrm{a}$ & $1,11 \mathrm{~b}$ & $0,0033 b$ & $182,61 \mathrm{a}$ \\
\hline \multicolumn{9}{|l|}{ VELOCIDADES } \\
\hline $1,11 \mathrm{~m} \mathrm{~s}^{-1}$ & $2,58 \mathrm{~d}$ & $0,0106 \mathrm{a}$ & $153,11 \mathrm{a}$ & $1,77 \mathrm{~b}$ & $3,28 \mathrm{c}$ & $1,55 \mathrm{a}$ & $0,0041 \mathrm{a}$ & $169,32 \mathrm{bc}$ \\
\hline $1,67 \mathrm{~m} \mathrm{~s}^{-1}$ & $2,94 \mathrm{c}$ & $0,0116 \mathrm{a}$ & $93,12 b$ & $1,81 \mathrm{~b}$ & $3,21 \mathrm{c}$ & $1,04 \mathrm{~b}$ & $0,0040 \mathrm{a}$ & $159,12 \mathrm{c}$ \\
\hline $2,22 \mathrm{~m} \mathrm{~s}^{-1}$ & $3,43 \mathrm{~b}$ & $0,0112 \mathrm{a}$ & $83,87 \mathrm{~b}$ & $2,11 \mathrm{a}$ & $3,92 \mathrm{~b}$ & $0,91 \mathrm{~b}$ & $0,0038 \mathrm{a}$ & $193,23 \mathrm{ab}$ \\
\hline $2,78 \mathrm{~m} \mathrm{~s}^{-1}$ & $3,93 \mathrm{a}$ & $0,0110 \mathrm{a}$ & $85,15 \mathrm{~b}$ & $2,09 \mathrm{a}$ & $4,32 \mathrm{a}$ & $0,87 \mathrm{~b}$ & $0,0043 \mathrm{a}$ & $194,27 \mathrm{a}$ \\
\hline \multicolumn{9}{|l|}{$\overline{\mathrm{CV}, \mathrm{DP} \text { e } \mathrm{MG}}$} \\
\hline$\overline{\mathrm{CV}(\%)}$ & 9,12 & 13,49 & 11,28 & 5,58 & 3,97 & 23,70 & 37,71 & 15,63 \\
\hline Desvio padrão (DP) & 0,64 & 0,00219 & 54,31 & 5,58 & 0,51 & 0,50 & 0,00191 & 38,77 \\
\hline Média geral (MG) & 3,22 & 0,0111 & 103,81 & 1,94 & 3,68 & 1,09 & 0,0040 & 178,99 \\
\hline \multicolumn{9}{|l|}{ Teste F } \\
\hline$\overline{\text { Sulcador (F1) }}$ & $4,33 *$ & $75,70 * *$ & $86,19 * *$ & $237,09 * *$ & $15,58 * *$ & $29,94 * *$ & $46,83^{* *}$ & $6,61 *$ \\
\hline Disco de corte (F2) & $22,77 * *$ & $4,89 *$ & $15,05^{* *}$ & $130,01 * *$ & $1,55 \mathrm{~ns}$ & $18,99 * *$ & $6,77 * *$ & $13,80 * *$ \\
\hline Velocidade (F3) & $72,66^{* *}$ & $1,41 \mathrm{~ns}$ & $28,52 * *$ & $51,12 * *$ & $237,98 * *$ & $26,50 * *$ & $0,36 \mathrm{~ns}$ & $7,09 * *$ \\
\hline $\mathrm{F} 1 \times \mathrm{F} 2$ & $0,71 \mathrm{~ns}$ & $0,47 \mathrm{~ns}$ & $3,71 *$ & $20,82 * *$ & $1,15 \mathrm{~ns}$ & $5,25^{* *}$ & $0,73 \mathrm{~ns}$ & $1,83 \mathrm{~ns}$ \\
\hline $\mathrm{F} 1 \times \mathrm{F} 3$ & $1,82 \mathrm{~ns}$ & $0,39 \mathrm{~ns}$ & $2,96^{*}$ & $4,84 * *$ & $8,48 * *$ & $3,33^{*}$ & $0,04 *$ & $0,08 \mathrm{~ns}$ \\
\hline $\mathrm{F} 2 \times \mathrm{F} 3$ & $1,81 \mathrm{~ns}$ & $0,62 \mathrm{~ns}$ & $5,06 * *$ & $9,05 * *$ & $5,18 * *$ & $5,31 * *$ & $0,35 \mathrm{~ns}$ & $0,89 \mathrm{~ns}$ \\
\hline $\mathrm{F} 1 \times \mathrm{F} 2 \times \mathrm{F} 3$ & $1,66 \mathrm{~ns}$ & $1,42 \mathrm{~ns}$ & $1,72 \mathrm{~ns}$ & $19,07 * *$ & $6,24 * *$ & $3,80 * *$ & $0,06 * *$ & $4,26 * *$ \\
\hline
\end{tabular}

Médias seguidas da mesma letra, na coluna, não diferem significativamente entre si, pelo teste de Tukey (p<0,05); ** Significativo à $1 \%$ de probabilidade de erro $(\mathrm{p}<0,01) ; *$ Significativo à $5 \%$ de probabilidade de erro $(\mathrm{p}<0,05)$; ns - Não significativo $(\mathrm{p}>0,05)$.

Os valores de patinamento encontrados mantiveram-se abaixo do considerado adequado pela ASABE (2006), tanto para solos firmes (8 a 10\%) como para mobilizados (11 a 13\%), obtendo-se o valor médio de 3,22\%. Este fato deve-se em virtude de que o esforço tratório necessário para tracionar o conjunto foi pequeno, por se tratar de um ensaio com apenas uma linha de interação com o solo. Foi observada influência significativa do tipo de sulcador sobre o patinamento, sendo que SF atingiu o maior valor, 3,29\%, enquanto o SR apresentou 3,15\%. Esta condição deve-se, principalmente, em virtude da ação diferenciada entre os mecanismos para a abertura do sulco, da profundidade de trabalho utilizada, por a haste ser 50,0\% maior que a dos discos e, devido a isso, demandar maior Ft. LEVIEN et al. (2011), trabalhando com diferentes mecanismos, também concluíram que abridores de sulco com órgão ativo fixo apresentam maior Pa que os rotativos.

Os discos de corte liso e ondulado não apresentaram diferença estatística entre si, sendo que apenas foi evidenciado contraste no patinamento, quando comparado à situação em que não foi utilizado nenhum organismo de corte à frente dos abridores de sulco. Confrontando com esta, o emprego deste tipo de mecanismo de corte proporcionou acréscimo no patinamento de 15,57\%, nas combinações que empregaram o liso, e de $18,34 \%$ para os que adotaram o ondulado. 
A interferência da velocidade sobre o patinamento foi significativamente diferente entre velocidades e proporcional ao aumento destas, sendo verificada a ampliação de $52,32 \%$ entre as velocidades de 1,11 e $2,78 \mathrm{~m} \mathrm{~s}^{-1}$. Tal fato deve-se, possivelmente, ao incremento no esforço de tração do conjunto. CHIODEROLLI et al. (2012), analisando o desempenho de um conjunto trator/semeadora, também evidenciaram acréscimo no patinamento com o incremento da velocidade de trabalho.

Foi observada influência do tipo de sulcador na Am, sendo que o sulcador fixo proporcionou a maior movimentação, com $0,0126 \mathrm{~m}^{2}$. Já os discos duplos moveram $31,25 \%$ menos que as hastes, com $0,0096 \mathrm{~m}^{2}$. Esta situação foi devida à maior profundidade de trabalho utilizada para a haste, pela ação diferenciada entre os mecanismos para a abertura do sulco e pelas diferenças entre as características dimensionais dos elementos que interferem nas tensões de corte, cisalhamento e compactação que estes provocam no solo. Este resultado está de acordo com o encontrado por MODOLO et al. (2012), que também concluíram que SF mobiliza mais o solo que o SR. Contudo, os valores encontrados foram superiores aos da literatura devido principalmente à menor umidade gravimétrica, reduzindo o efeito lubrificante da água e, por consequência, proporcionando maior mobilização, além de outros contrastes entre características físicas do solo, como densidade e resistência à penetração, e pelas diferenças técnicas entre sulcadores utilizados.

Os discos de corte apresentaram diferença estatística na Am, sendo que o disco liso mobilizou $12,26 \%$ a menos que o disco ondulado, devido às diferenças do projeto dos elementos e à maior área de contato com o solo deste último. Além disso, a presença deste, quando comparada com a situação sem a utilização de qualquer disco, também acarretou maior Am. Contudo, quando se fez uso do disco liso nesta mesma equiparação, não foi encontrado contraste. MION et al. (2009), trabalhando com diferentes discos de corte de palhada, também encontraram diferenças estatísticas entre a Am, sendo que o disco ondulado movimentou mais que o liso.

O emprego de diferentes velocidades não influenciou a Am, sendo que esta apresentou média de 0,0111 $\mathrm{m}^{2}$. Estes resultados estão de acordo com os encontrados por BELLÉ et al. (2014).

Foi observada diferença estatística entre os diferentes sulcadores sobre o consumo específico de combustível, sendo que o rotativo apresentou consumo 76,73\% superior ao fixo, ficando em $132,60 \mathrm{~g} \mathrm{kWh}^{-1} \mathrm{~m}^{-2}$ para os discos duplos e 75,03 $\mathrm{g} \mathrm{kWh}^{-1} \mathrm{~m}^{-2}$ para a haste.

A utilização de discos de corte à frente dos sulcadores reduziu o consumo específico em $48,57 \%$, para a combinação com disco liso, e em $25,65 \%$, para as que utilizaram disco ondulado. Por sua vez, não foi observada diferença estatística entre os resultados obtidos nos diferentes discos.

Nas velocidades de 1,$67 ; 2,22$ e $2,78 \mathrm{~m} \mathrm{~s}^{-1}$, não foi observada variação no consumo específico operacional. Contudo, estes resultados foram estatisticamente diferentes para a situação onde foi utilizada a velocidade de $1,11 \mathrm{~m} \mathrm{~s}^{-1}$. Tal fato deve-se em função de que a menor força de tração, consequentemente a potência demandada, foi obtida nesta velocidade, associada à manutenção da área mobilizada nas diferentes velocidades.

A força de tração demandada em função da associação entre sulcador e disco de corte, nas diferentes velocidades de deslocamento, bem como a média obtida para cada interação entre mecanismos, está disposta na Tabela 2. 
TABELA 2. Valores médios da força de tração para as interações entre sulcador, disco de corte e velocidade. Traction averages for the furrow, cutting disk and speed interactions.

\begin{tabular}{ccccccc}
\hline \multirow{2}{*}{ Interação Sulcador/Disco } & \multicolumn{4}{c}{ Velocidade de deslocamento $\left(\mathrm{m} \mathrm{s}^{-1}\right)$} & \multirow{2}{*}{ Média (kN) } \\
\cline { 2 - 5 } & & 1,11 & 1,67 & 2,22 & 2,78 & \\
\hline SF & SD & $1,42 \mathrm{cB}$ & $1,26 \mathrm{cB}$ & $2,22 \mathrm{bA}$ & $2,38 \mathrm{abA}$ & 1,82 \\
SF & $\mathrm{DL}$ & $2,08 \mathrm{aA}$ & $2,14 \mathrm{aA}$ & $2,22 \mathrm{bA}$ & $2,19 \mathrm{bcA}$ & 2,16 \\
$\mathrm{SF}$ & $\mathrm{DO}$ & $2,32 \mathrm{aB}$ & $2,40 \mathrm{aAB}$ & $2,58 \mathrm{aA}$ & $2,47 \mathrm{aAB}$ & 2,44 \\
$\mathrm{SR}$ & $\mathrm{SD}$ & $1,47 \mathrm{bcA}$ & $1,44 \mathrm{cA}$ & $1,50 \mathrm{dA}$ & $1,56 \mathrm{eA}$ & 1,49 \\
$\mathrm{SR}$ & $\mathrm{DL}$ & $1,69 \mathrm{bB}$ & $1,82 \mathrm{bB}$ & $2,30 \mathrm{bA}$ & $1,89 \mathrm{~dB}$ & 1,92 \\
$\mathrm{SR}$ & $\mathrm{DO}$ & $1,62 \mathrm{bcB}$ & $1,76 \mathrm{bB}$ & $1,83 \mathrm{cB}$ & $2,08 \mathrm{cdA}$ & 1,82 \\
\hline Média & & 1,77 & 1,80 & 2,11 & 2,09 & - \\
\hline
\end{tabular}

Médias seguidas da mesma letra, minúscula na coluna e maiúscula na linha, não diferem significativamente entre si, pelo teste de Tukey $(\mathrm{p}<0,05)$.

O requerimento médio de tração do sulcador fixo foi $22,28 \%$ superior ao solicitado pelo mecanismo rotativo. $\mathrm{O}$ primeiro necessitou de $2,14 \mathrm{kN}$, enquanto o segundo demandou $1,75 \mathrm{kN}$. LEVIEN et al. (2011), avaliando a utilização de discos duplos em um Nitossolo Vermelho distroférrico de textura muito argilosa, encontraram valores médios de 1,93 kN, e ALMEIDA, SILVA \& SILVA (2010), trabalhando com hastes sulcadoras, evidenciaram demanda de 2,34 kN por linha de semeadura. Assim sendo, os resultados encontrados estão condizentes com os obtidos pela literatura. Além disso, LEVIEN et al. (2011), após terem avaliado diferentes sulcadores, também evidenciaram que elementos fixos proporcionam maior exigência de tração. Isso pode ser explicado em função de que o projeto da haste visa a quebrar as camadas mais compactadas, trabalhando a maior profundidade, o que proporciona maior demanda de energia para vencer $o$ acréscimo de atrito entre a ferramenta e solo, ao contrário dos discos duplos, que apenas abrem o sulco.

A situação sem disco de corte foi a que demandou a menor força de tração, apresentando diferença significativa de 22,89 e $28,31 \%$ para os discos liso e ondulado, respectivamente. Além disso, comparando entre os mecanismos de corte, o ondulado foi o que exigiu maior esforço de tração, apresentando diferença de $4,41 \%$ para o liso. Isso é justificado em função de que estes apresentam resistência ao rolamento, de forma que a presença de discos aumenta a solicitação de tração e, por consequência da maior superfície específica, ou seja, maior área de conta to com o solo, o ondulado é o que propiciou os maiores requerimentos.

Com relação à variável velocidade, as duas mais lentas, 1,11 e $1,67 \mathrm{~m} \mathrm{~s}^{-1}$, não apresentaram contraste na exigência de tração, assim como entre as mais rápidas, 2,22 e 2,78 $\mathrm{m} \mathrm{s}^{-1}$. Contudo, foi evidenciada influência significativa sobre a demanda entre estes grupos, sendo que o primeiro foi o que exigiu menor força, apresentando diferença de 17,32\%. Este fato foi comprovado por FURLANI et al. (2013), avaliando a demanda de tração de uma semeadora em diferentes velocidades.

$\mathrm{Na}$ velocidade menor, foi observado que as combinações SR SD e SR DO não apresentaram diferença significativa para a SF SD. Na condição de $1,67 \mathrm{~m} \mathrm{~s}^{-1}$, essa igualdade foi observada entre SF SD e SR SD, e em 2,22 $\mathrm{m} \mathrm{s}^{-1}$, entre SF DL e SR DO. Já para a maior velocidade, não houve igualdade na demanda entre o SF e o SR em nenhuma combinação. Desta forma, em determinadas associações entre velocidade e discos de corte para o SR, a exigência por parte destes pode ser semelhante ao SF, desde que estes últimos trabalhem em baixas velocidades e sem emprego de discos.

Além disso, foi evidenciado que a utilização de discos de corte, quando associada ao sulcador fixo, reduziu a ampliação da demanda de tração em aproximadamente $60 \%$, quando a velocidade passou de 1,11 para $2,78 \mathrm{~m} \mathrm{~s}^{-1}$. Na condição sem disco, a ampliação de tração entre estas velocidades foi de $67,61 \%$, enquanto, quando combinada com o disco liso, este acréscimo foi de apenas $5,28 \%$ e de $6,46 \%$ quando associado ao ondulado. 
O consumo horário de combustível $(\mathrm{Ch})$ apresentou influência dos mecanismos de corte, dos abridores de sulco e da velocidade de deslocamento. Os resultados desta interação são apresentados na Tabela 3.

TABELA 3. Valores médios do consumo horário de combustível (Ch) para as interações entre sulcador, disco de corte e velocidade. Hourly fuel consumption (Ch) averages for furrow, cutting disk and speed interactions.

\begin{tabular}{ccccccc}
\hline \multirow{2}{*}{ Interação Sulcador/Disco } & \multicolumn{3}{c}{ Velocidade de des locamento $\left(\mathrm{m} \mathrm{s}^{-1}\right)$} & \multirow{2}{c}{ Ch } \\
\cline { 2 - 5 } & & 1,11 & 1,67 & 2,22 & 2,78 & Média $\left(\mathrm{L} \mathrm{h}^{-1}\right)$ \\
\hline SF & SD & $3,49 \mathrm{aB}$ & $3,53 \mathrm{aB}$ & $4,05 \mathrm{abA}$ & $4,24 \mathrm{bA}$ & 4,08 \\
SF & DL & $3,28 \mathrm{aB}$ & $2,94 \mathrm{cC}$ & $4,30 \mathrm{aA}$ & $4,26 \mathrm{abA}$ & 3,70 \\
SF & DO & $3,29 \mathrm{aC}$ & $3,28 \mathrm{abcC}$ & $4,00 \mathrm{abB}$ & $4,35 \mathrm{abA}$ & 3,73 \\
SR & SD & $3,17 \mathrm{aC}$ & $3,11 \mathrm{bcC}$ & $3,87 \mathrm{bB}$ & $4,35 \mathrm{abA}$ & 3,62 \\
SR & DL & $3,21 \mathrm{aB}$ & $3,34 \mathrm{abB}$ & $3,82 \mathrm{bA}$ & $4,12 \mathrm{bA}$ & 3,62 \\
SR & DO & $3,23 \mathrm{aBC}$ & $3,08 \mathrm{bcC}$ & $3,46 \mathrm{cB}$ & $4,62 \mathrm{aA}$ & 3,60 \\
\hline Ch - Média $\left(\mathrm{L} \mathrm{h}^{-1}\right)$ & & 3,44 & 3,21 & 3,92 & 4,32 & - \\
\hline
\end{tabular}

Médias seguidas da mesma letra, minúscula na coluna e maiúscula na linha, não diferem significativamente entre si, pelo teste de Tukey $(\mathrm{p}<0,05)$.

Foi evidenciado que o sulcador fixo apresentou maior consumo de combustível que o rotativo, sendo esta diferença de 3,88\%. Esta situação deve-se ao maior requerimento de tração demandada por este tipo de mecanismo. Estes resultados estão de acordo com os obtidos por LEVIEN et al. (2011), que avaliaram a demanda energética de uma semeadora em sistema de semeadura direta.

Não foi observada influência do fator disco de corte sobre o $\mathrm{Ch}$, sendo que este apresentou gasto médio de 3,68 $\mathrm{L} \mathrm{h}^{-1}$. MAHL et al. (2007) também observaram este desempenho, obtendo um valor por linha de semeadura de $3,47 \mathrm{~L} \mathrm{~h}^{-1}$, em um solo arenoso, e de $3,10 \mathrm{~L} \mathrm{~h}^{-1}$, em um argiloso.

Ocorreu incremento de $31,71 \%$ no consumo de combustível com o acréscimo da velocidade de 1,11 para $2,78 \mathrm{~m} \mathrm{~s}^{-1}$. Contudo, não foi observada diferença estatística nesta variável entre as velocidades mais baixas. Estas apresentaram uma diferença média de 20,49\%, em comparação à velocidade de $2,22 \mathrm{~m} \mathrm{~s}^{-1}$, e de $33,13 \%$ quando se fez uso de 2,78 $\mathrm{m} \mathrm{s}^{-1}$. SILVEIRA et al. (2013), avaliando as exigências de uma semeadora em função da velocidade de deslocamento, identificaram acréscimo do consumo horário quando passou a velocidade de 1,11 para $1,67 \mathrm{~m} \mathrm{~s}^{-1}$.

Foi verificado que não ocorreu diferença significativa no $\mathrm{Ch}$ entre todas as combinações sulcador/disco na velocidade de $1,11 \mathrm{~m} \mathrm{~s}^{-1}$. Além disso, as associações SF SD, SF DO e SR DL foram as que mais consumiram na velocidade de $1,67 \mathrm{~m} \mathrm{~s}^{-1}$ e as interações com SF na velocidade $2,22 \mathrm{~m} \mathrm{~s}^{-1}$. Ademais, em 2,78 $\mathrm{m} \mathrm{s}^{-1}$, foram as composições SF DL, SF DO, SR SD e SR DO.

O consumo específico $(\mathrm{Ce})$ apresentou interação entre o fator disco de corte e a velocidade de deslocamento. Os resultados desta avaliação estão apresentados na Tabela 4.

$\mathrm{O}$ sulcador rotativo foi o elemento abridor de sulco que apresentou o maior $\mathrm{Ce}$, exibindo a diferença média de $0,34 \mathrm{~g} \mathrm{kWh}^{-1}$, representando $36,96 \%$ para o sulcador fixo.

$\mathrm{O}$ fator disco de corte apresentou influência significativa sobre o $\mathrm{Ce}$, sendo que a situação sem a utilização destes mecanismos foi a que proporcionou o maior consumo, seguido pelo ondulado e pelo liso. A condição sem disco apresentou diferença de 18,02 e 54,12\% para os discos liso e ondulado, respectivamente. 
TABELA 4. Valores médios do consumo específico de combustível (Ce) para as interações entre sulcador, disco de corte e velocidade. Specific fuel cons umption (Ce) averages for furrow, cutting disk and speed interactions.

\begin{tabular}{|c|c|c|c|c|c|c|}
\hline \multirow{2}{*}{\multicolumn{2}{|c|}{ Interação Sulcador/Disco }} & \multicolumn{4}{|c|}{ Velocidade de deslocamento $\left(\mathrm{m} \mathrm{s}^{-1}\right)$} & \multirow{2}{*}{$\begin{array}{l}\text { Ce - Média } \\
\left(\mathrm{g} \mathrm{kWh}^{-1}\right)\end{array}$} \\
\hline & & 1,11 & 1,67 & 2,22 & 2,78 & \\
\hline SF & SD & $1,98 \mathrm{aA}$ & $1,49 \mathrm{aA}$ & $0,85 \mathrm{bB}$ & $0,61 \mathrm{bB}$ & 1,23 \\
\hline SF & DL & $1,17 \mathrm{bA}$ & $0,66 \mathrm{bAB}$ & $0,67 \mathrm{bAB}$ & $0,40 \mathrm{bB}$ & 0,72 \\
\hline SF & DO & $1,19 \mathrm{bA}$ & $0,79 \mathrm{bAB}$ & $0,59 \mathrm{bB}$ & $0,66 \mathrm{bAB}$ & 0,81 \\
\hline SR & SD & $1,76 \mathrm{abA}$ & $1,17 \mathrm{abBC}$ & $1,69 \mathrm{aAB}$ & $0,90 \mathrm{bC}$ & 1,38 \\
\hline SR & DL & $1,51 \mathrm{abA}$ & $0,97 \mathrm{abAB}$ & $0,67 \mathrm{bB}$ & $0,73 \mathrm{bB}$ & 0,97 \\
\hline SR & DO & $1,67 \mathrm{abAB}$ & $1,16 \mathrm{abBC}$ & $0,95 \mathrm{bC}$ & $1,89 \mathrm{aA}$ & 1,42 \\
\hline$\overline{C e}-$ Médi & & 1,55 & 1,04 & 0,90 & 0,86 & - \\
\hline
\end{tabular}

Médias seguidas da mesma letra, minúscula na coluna e maiúscula na linha, não diferem significativamente entre si pelo teste de Tukey $(\mathrm{p}<0,05)$.

O consumo específico foi maior na velocidade de $1,11 \mathrm{~m} \mathrm{~s}^{-1}$, sendo que esta apresentou diferença significativa para as demais, e estas não diferiram entre si. A primeira divergiu em $49,04 \%$ para a velocidade de $1,67 \mathrm{~m} \mathrm{~s}^{-1}, 70,33 \%$ para a condição onde se fez uso da velocidade de $2,22 \mathrm{~m} \mathrm{~s}^{-1}$ e $78,16 \%$ para a 2,78 $\mathrm{m} \mathrm{s}^{-1}$. Tal fato pode ser devido a que o aumento da velocidade proporciona maior incremento da demanda da potência do que do acréscimo no consumo horário. Como resultado, verificou-se melhor aproveitamento da energia liberada pelo combustível nas maiores velocidades, já que foi necessário menor massa para originar a mesma quantidade de energia.

As associações entre SF SD, SR SD, SR DL e SR DO foram as que apresentaram o maior consumo na velocidade de $1,11 \mathrm{~m} \mathrm{~s}^{-1}$. Ademais, com exceção da combinação SR DO, foram encontrados, sem diferença estatística entre as combinações, os menores resultados de consumo específico de combustível na velocidade de $2,78 \mathrm{~m} \mathrm{~s}^{-1}$.

A interação entre sulcador, disco de corte e a velocidade de deslocamento sobre a área elevada (Ae) está disposta na Tabela 6.

TABELA 6. Valores médios da área elevada (Ae) para as interações entre sulcador, disco de corte e velocidade. Elevated área (Ae) averages for furrow, cutting disk and speed interactions.

\begin{tabular}{ccccccc}
\hline \multirow{2}{*}{ Interação Sulcador/Disco } & \multicolumn{4}{c}{ Velocidade de deslocamento $\left(\mathrm{m} \mathrm{s}^{-1}\right)$} & $\begin{array}{c}\text { Ae } \\
\text { Média }\left(\mathrm{m}^{2}\right)\end{array}$ \\
\cline { 2 - 6 } & SD & 1,11 & 1,67 & 2,22 & 2,78 & M \\
SF & DL & $0,0045 \mathrm{aA}$ & $0,0054 \mathrm{aA}$ & $0,0060 \mathrm{aA}$ & $0,0060 \mathrm{aA}$ & 0,0059 \\
SF & DO & $0,0053 \mathrm{abA}$ & $0,0052 \mathrm{aA}$ & $0,0049 \mathrm{abA}$ & $0,0056 \mathrm{abA}$ & 0,0050 \\
SF & SD & $0,0042 \mathrm{abA}$ & $0,0033 \mathrm{aA}$ & $0,0045 \mathrm{abA}$ & $0,0048 \mathrm{abA}$ & 0,0048 \\
SR & DL & $0,0025 \mathrm{bA}$ & $0,0029 \mathrm{aA}$ & $0,0027 \mathrm{abA}$ & $0,0039 \mathrm{abA}$ & 0,0038 \\
SR & DO & $0,0016 \mathrm{bA}$ & $0,0020 \mathrm{aA}$ & $0,0012 \mathrm{bA}$ & $0,0021 \mathrm{bA}$ & 0,0017 \\
SR & 0,0041 & 0,0039 & 0,0038 & 0,0043 & - \\
\hline Ae - Média $\left(\mathrm{m}^{2}\right)$ & & &
\end{tabular}

Médias seguidas da mesma letra, minúscula na coluna e maiúscula na linha, não diferem significativamente entre si, pelo teste de Tukey $(\mathrm{p}<0,05)$.

Foi observada a redução de $89,28 \%$ da Ae quando se fez uso do sulcador rotativo em comparação à utilização do fixo. O primeiro elemento elevou $0,0028 \mathrm{~m}^{2}$, enquanto o segundo foi responsável por $0,0052 \mathrm{~m}^{2}$. Isto é devido, possivelmente, por os discos duplos estarem dispostos em menor profundidade de trabalho que a haste, resultando em menor Ae, assim como pela ação de corte e não de cisalhamento promovida por estes para a abertura de sulco. 
Não foi encontrada diferença estatística entre a área elevada promovida pelos discos liso e ondulado. Contudo, foi observado contraste quando comparado este último com a situação sem a presença destes mecanismos, sendo que o ondulado elevou 48,48\% menos. Esta situação pode ser em decorrência do maior fracionamento do solo proporcionado por este tipo de mecanismo, em comparação à situação sem disco, por consequência da maior área de contato entre elemento e solo.

O emprego de discos de corte apenas apresentou diferença estatística na elevação do solo, quando comparado à associação entre disco ondulado e sulcador rotativo com a combinação sulcador fixo sem disco, nas velocidades de deslocamento de 2,22 e $2,78 \mathrm{~m} \mathrm{~s}^{-1}$. Além disso, na velocidade de $1,11 \mathrm{~m} \mathrm{~s}^{-1}$, foi observada distinção entre o SR e o disco liso com a haste sem disco. Em ambas as situações, o emprego do sulcador fixo apresentou a maior elevação de solo. Nas demais situações, não foram evidenciadas diferenças entre os mecanismos, principalmente na velocidade de 1,67 $\mathrm{m} \mathrm{s}^{-1}$, em que nenhuma combinação apresentou diferença na elevação de solo.

Foi observada influência de todos os fatores sobre a resistência específica (Reo), sendo que os resultados desta são apresentados na Tabela 7.

TABELA 7. Valores médios da resistência específica (Reo) para as interações entre sulcador, disco de corte e velocidade. Specific resistance (Reo) averages for furrow, cutting disk and speed interactions.

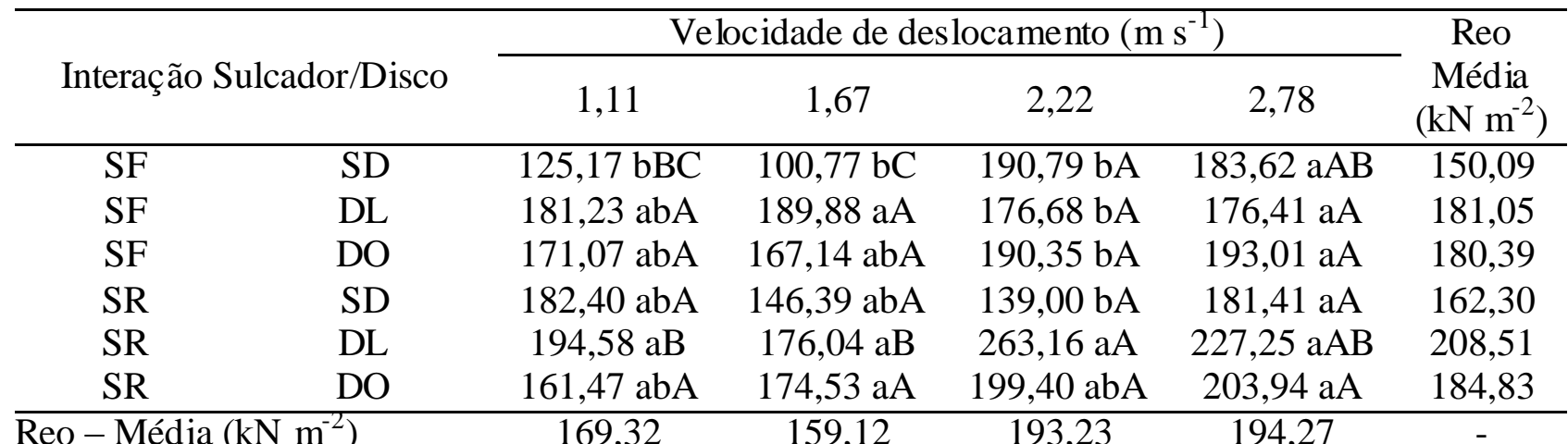

Médias seguidas da mesma letra, minúscula na coluna e maiúscula na linha, não diferem significativamente entre si, pelo teste de Tukey $(\mathrm{p}<0,05)$.

A necessidade de tração por área de solo mobilizado dos sulcadores rotativos apresentou 9,94\% maior do que a haste sulcadora. Este fato foi devido à maior demanda de tração e área mobilizada propiciada por estes elementos.

A presença de DC proporcionou incremento na Reo em comparação à situação sem o emprego destes elementos. O DL apresentou acréscimo de $26,86 \%$, e o DO, de $16,91 \%$, em comparação a condição sem disco. Ademais, não foi verificada diferença significativa entre estes mecanismos.

O incremento da velocidade propiciou aumento significativo sobre a Reo, corroborando com os resultados de GASSEN et al. (2014), ao estudar a operação de escarificação. Nas mais altas, 2,22 e $2,78 \mathrm{~m} \mathrm{~s}^{-1}$, não foi observada diferença significativa entre ambas. Além disso, a primeira também não diferiu de $1,11 \mathrm{~m} \mathrm{~s}^{-1}$, e esta de $1,67 \mathrm{~m} \mathrm{~s}^{-1}$. Isso pode ser explicado em virtude da maior $\mathrm{Ft}$ requerida pelas combinações nas velocidades mais elevadas, já que não houve incremento da Am.

As associações entre o sulcador fixo e os discos liso e ondulado, além do sulcador rotativo sem disco e com disco ondulado, não apresentaram influência da velocidade sobre a resistência específica. Por outro lado, as combinações de haste sulcadora sem disco e discos duplos com disco liso proporcionaram incremento da variável em função do aumento da velocidade. Além disso, a haste sulcadora sem a utilização de nenhum disco de corte foi a combinação, na velocidade de 1,11 e $1,67 \mathrm{~m} \mathrm{~s}^{-1}$, que apresentou o menor valor de resistência, com 125,17 e $100,77 \mathrm{kN} \mathrm{m}^{-2}$, respectivamente. $\mathrm{Na}$ velocidade seguinte, $2,22 \mathrm{~m} \mathrm{~s}^{-1}$, as combinações que empregaram o sulcador fixo e a condição do sulcador rotativo sem disco, foram as que obtiveram os menores valores de 
demanda de tração por área de solo mobilizada. Já na velocidade de $2,78 \mathrm{~m} \mathrm{~s}^{-1}$, não foi verificada diferença estatística entre as diferentes combinações de sulcador/disco.

\section{CONCLUSÕES}

O sulcador tipo haste exigiu maior força de tração que os de discos duplos desencontrados, e ambos, ao serem combinados com discos de corte, tiveram seus requerimentos aumentados.

Houve a proporcionalidade da Ft sobre o $\mathrm{Ch}$ e o $\mathrm{Pa}$, que aumentaram com a velocidade e foram maiores com o uso de hastes em relação aos discos duplos. Já o Ce e o Ceo foram inversamente proporcionais à $\mathrm{Ft}$, sendo maior para as associações com o SR. As variáveis de solo não foram influenciadas pela velocidade, sendo maior com o uso de haste. Dentre as combinações com disco de corte, o ondulado propiciou a maior Am e a menor Ae.

A Reo aumentou com a velocidade, sendo maior para o sulcador rotativo. Dessa forma, a haste foi mais eficiente no uso da tração por unidade de solo mobilizado.

Não se recomenda a utilização das velocidades 2,22 e $2,78 \mathrm{~m} \mathrm{~s}^{-1}$, independentemente da associação sulcador/disco, devido a estas apresentarem maior Ft e Ch.

\section{REFERÊNCIAS}

ALMEIDA, R. A. S.; SILVA, C. A. T.; SILVA, S. de L. Desempenho energético de um conjunto trator-semeadora em função do escalonamento de marchas e rotações do motor. Revista Agrarian, Dourados, v. 3, n.7, p. 63-70, 2010.

ASABE - AMERICAN SOCIETY OF AGRICULTURAL AND BIOLOGICAL ENGINEERS. Agricultural machinery management. St. Joseph: EP 496.3, 2006.

BELlÉ, M. P.; ALONÇO, A. dos S.; FRANCETTO, T. R.; ROSSATO, F. P.; FRANCK, C. J.; CARPES, D. P. Demanda energética e mobilização do solo com o uso de escarificadores em sistemas de semeadura direta. Revista Brasileira de Engenharia Agrícola e Ambiental, Campina Grande, v.18, n. 5, p. 551-558, 2014.

CHIODEROLI, C. A.; FURLANI, C. E. A.; AGUIAR, A. O.; CAVICHIOLI, F. A.; CASSIA, M.T. Operational parameters of soybean seeding in santa fe system. Engenharia Agrícola, Jaboticabal, v. 32, n. 5, p. 900-908, 2012.

EMBRAPA - EMPRESA BRASILEIRA DE PESQUISA AGROPECUÁRIA. Manual de métodos de análise de solo. Rio de Janeiro, 1997. 212 p.

FURLANI, C. E. A.; CANOVA, R.; CAVICHIOLI, F. A.; BERTONHA, R. S.; SILVA, R. P. Demanda energética por semeadora-adubadora em função da haste sulcadora na semeadura do milho. Revista Ceres, Viçosa, MG, v. 60, n. 6, p. 885-889. 2013.

GASSEN, J. R. F; ALONÇO, A. dos S.; BAUMHARDT, U. B.; BELlÉ, M. P.; BONOTTO, G. J. Resistência específica à tração na operação de escarificação do solo em camadas de forma simultânea. Revista Brasileira de Engenharia Agrícola e Ambiental, Campina Grande, v. 18, n. 1, p. 116-124, 2014.

IBGE - INSTITUTO BRASILEIRO DE GEOGRAFIA E ESTATÍSTICA. Indicadores IBGE: estatísticas da produção agrícola. 2013. Disponível em: < http://www.ibge.gov.br/home/estatistica/indicadores/agropecuaria/lspa/lspa_201301comentarios.pdf >. Acesso em: 28 abr. 2014.

LEVIEN, R.; FURLANI, C. E. A.; GAMERO; C. A.; CONTE, O.; CAVICHIOLI, F. A. Semeadura direta de milho com dois tipos de sulcadores de adubo, em nível e no sentido do declive do terreno. Ciência Rural, Santa Maria, v. 41, n. 6, p. 1003-1010, 2011. 
MAHL, D., GAMERO, C. A., BENEZ, S. H. Demanda energética de semeadora-adubadora de plantio direto em função de elementos de corte, velocidade e tipo de solo. Energia na Agricultura, Botucatu, v. 22, n. 3, p. 15-36, 2007.

MIALHE, L. G. Máquinas agrícolas para plantio. Campinas, 2012. 623 p.

MION, R. L.; BENEZ, S. H.; VILIOTTI, C. A.; MOREIRA, J. B.; SALVADOR, N.. Análise tridimensional de esforços em elementos rompedores de semeadoras de plantio direto. Ciência Rural, Santa Maria, v. 39, n. 5, p. 1414-1419, 2009.

MODOlO, A. J.; TROGEllo, E.; PAGliosA, E. S.; DALlACORT, R.; KOLlinG, E. M.; SGARBOSSA, M.. Seeding quality and soybean yields from using different furrowers and operation speeds. Semina: Ciências Agrárias, Londrina, v. 33, supl. 1, p. 3009-3016, 2012.

SILVEIRA, J. C. M.; FERNANDES, H. C.; MODOLO, A. J.; SILVA, S. de L.; TROGELlO, E. Demanda energética de uma semeadora-adubadora em diferentes velocidades de deslocamento e rotações do motor. Revista Ciência Agronômica, Fortaleza, v. 44, n. 1, p. 44-52, 2013. 